JOURNAL OF

SYMPLECTIC GEOMETRY

Volume 1, Number 3, 523-542, 2002

\title{
A classification of topologically stable Poisson structures on a compact oriented surface
}

\author{
Olga RADKO
}

\begin{abstract}
Poisson structures vanishing linearly on a set of smooth closed disjoint curves are generic in the set of all Poisson structures on a compact connected oriented surface. We construct a complete set of invariants classifying these structures up to an orientation-preserving Poisson isomorphism. We show that there is a set of non-trivial infinitesimal deformations which generate the second Poisson cohomology and such that each of the deformations changes exactly one of the classifying invariants. As an example, we consider Poisson structures on the sphere which vanish linearly on a set of smooth closed disjoint curves.
\end{abstract}

\section{Introduction.}

Recently several results were obtained concerning the local classification of Poisson structures on a manifold. According to the Splitting Theorem [11], the problem of local classification can be reduced to the classification of structures vanishing at a point. In dimension 2, V. Arnold [1] obtained a hierarchy of normal forms of germs of Poisson structures degenerate at a point (see also a paper by P. Monnier [6] for a detailed exposition.) Using the notion of the modular vector field of a Poisson structure, J.-P. Dufour and A. Haraki [2] and Z.-J. Liu and P. Xu [5] obtained a complete local classification of quadratic Poisson structures in dimension 3. Some results related to local classification of Poisson structures in dimensions 3 and 4 were also obtained by J. Grabowski, G. Marmo and A.M. Perelomov in [4].

However, not much is known in relation to the global classification of Poisson structures on a given manifold (i.e., classification up to a Poisson isomorphism, see [13] for a general discussion). In this paper we give an explicit example of a global classification of a certain set of Poisson structures on a compact oriented surface $\Sigma$.

For a compact connected oriented surface $\Sigma$ and $n \geq 0$ we consider the set $\mathcal{G}_{n}(\Sigma)$ of Poisson structures on $\Sigma$ which vanish linearly on a set of $n$ smooth simple closed non-intersecting (in short, disjoint) curves. In 
particular, $\mathcal{G}_{0}(\Sigma)$ is the space of symplectic structures. The set $\mathcal{G}(\Sigma) \doteq$ $\bigsqcup_{n>0} \mathcal{G}_{n}(\Sigma)$ is dense in the vector space $\Pi(\Sigma)$ of all Poisson structures on $\Sigma$. We call Poisson structures in $\mathcal{G}(\Sigma)$ topologically stable since the topology of their zero sets is unchanged under small perturbations. Locally around a point on a zero curve these structures have the first order of degeneracy in the Arnold's classification.

The main result of this paper is a complete classification of the topologically stable structures $\mathcal{G}(\Sigma)$ up to an orientation-preserving Poisson isomorphism. We construct the set of invariants of a structure $\pi \in \mathcal{G}_{n}(\Sigma)$ which consists of

- the topological arrangement of the zero curves $\gamma_{1}, \ldots, \gamma_{n}$ of $\pi$ taken with orientations defined by $\pi$ (up to an orientation-preserving diffeomorphism);

- the periods of the restriction of a modular vector field $X^{\omega_{0}}(\pi)$ with respect to a volume form $\omega_{0}$ to the zero curves of $\pi$;

- the volume invariant of $\pi$ generalizing the Liouville volume for a symplectic structure.

We prove that two structures $\pi, \pi^{\prime} \in \mathcal{G}_{n}(\Sigma)$ are globally equivalent via an orientation-preserving Poisson isomorphism if and only if all the invariants for these structures coincide.

The question of global equivalence of Poisson structures by orientationreversing Poisson isomorphisms can be reduced to the orientation-preserving case. Let $\nu: \Sigma \rightarrow \Sigma$ be an orientation-reversing diffeomorphism of $\Sigma$. Two Poisson structures $\pi, \pi^{\prime} \in \mathcal{G}_{n}(\Sigma)$ are globally equivalent via an orientation-reversing diffeomorphism iff $\nu_{*} \pi$ and $\pi^{\prime}$ are globally equivalent via an orientation-preserving diffeomorphism.

We compute the Poisson cohomology of a given Poisson structure $\pi \in$ $\mathcal{G}_{n}(\Sigma)$ on a compact oriented surface $\Sigma$ of genus $g$. The zeroth cohomology (interpreted as the space of Casimir functions) is generated by constant functions and is one-dimensional. The first cohomology (interpreted as the space of Poisson vector fields modulo Hamiltonian vector fields) has dimension $2 g+n$ and is generated by the image of the first de Rham cohomology of $\Sigma$ under the injective homomorphism $\tilde{\pi}: H^{*}(\Sigma) \rightarrow H_{\pi}^{*}(\Sigma)$ and by the following $n$ vector fields:

$$
X^{\omega_{0}}(\pi) \cdot\left\{\text { bump function around } \gamma_{i}\right\}, \quad i=1, \ldots n \text {. }
$$


(The map $\tilde{\pi}$ on the level of cohomology is induced by the canonical bundle map $\tilde{\pi}: T^{*} \Sigma \rightarrow T \Sigma$ associated to the Poisson structure $\pi$ in the following way: $\beta(\tilde{\pi}(\alpha))(x) \doteq \pi(\alpha, \beta)(x)$, where $\alpha, \beta \in \Gamma\left(T^{*} \Sigma\right), x \in \Sigma$.)

The second cohomology is generated by a non-degenerate Poisson structure $\pi_{0}$ on $\Sigma$ and $n$ Poisson structures of the form

$$
\pi_{i}=\pi \cdot\left\{\text { bump function around } \gamma_{i}\right\}, \quad i=1, \ldots, n .
$$

Each of the generators of the second cohomology corresponds to a oneparameter family of infinitesimal deformations of the Poisson structure which affects exactly one of the numeric classifying invariants. The deformation $\pi \mapsto \pi+\varepsilon \cdot \pi_{0}$ changes the regularized Liouville volume. For each $i=1, \ldots, n$ the deformation $\pi \mapsto \pi+\varepsilon \cdot \pi_{i}$ changes the modular period around the curve $\gamma_{i}$. This shows that the number of numeric classifying invariants $\left((n+1)\right.$ for $\left.\mathcal{G}_{n}(\Sigma)\right)$ equals to the dimension of the second Poisson cohomology, and is, therefore, optimal.

As an example we consider the topologically stable Poisson structures $\mathcal{G}\left(S^{2}\right)$ on the sphere. In this case, an explicit description of the moduli space of topologically stable Poisson structures up to an orientation-preserving Poisson isomorphism is obtained.

Since topologically stable Poisson structures considered in the present paper have degeneracies of the simplest kind, our work can be considered as a first step in the direction of global classification of Poisson structures having higher-order degeneracies in terms of the Arnold's hierarchy of germs of Poisson structures. We plan to pursue this direction in our future work.

Acknowledgement. I would like to thank my advisor, Prof. A.Weinstein, for proposing this problem and for many fruitful discussions.

\section{Invariants of topologically stable Poisson structures.}

First we recall the classification of symplectic structures on compact oriented surfaces which follows from Moser's theorem.

\subsection{Classification of symplectic structures.}

According to Darboux's theorem, all symplectic structures on a given manifold $M$ are locally equivalent: for each symplectic form $\omega$ and a point $p \in M^{2 n}$, there exist a coordinate system $\left(U, x_{1}, \ldots, x_{n}, y_{1}, \ldots, y_{n}\right)$ centered at $p$ such that $\omega=\sum_{i=1}^{n} d x_{i} \wedge d y_{i}$ on $U$. Therefore, the dimension of the underlying manifold is the only local invariant of a symplectic structure. 
Definition 1. Two symplectic forms $\omega$ and $\omega^{\prime}$ on $M$ are globally equivalent if there is a symplectomorphism $\varphi:(M, \omega) \rightarrow\left(M, \omega^{\prime}\right)$.

In certain cases the following theorem of Moser allows one to classify symplectic forms on a given manifold up to global equivalence:

Theorem 1 (Moser, [7]). Let $\omega$ and $\omega^{\prime}$ be symplectic forms on a compact connected manifold $M$. Suppose that $[\omega]=\left[\omega^{\prime}\right] \in H^{2}(M)$ and that the 2form $\omega_{t} \doteq(1-t) \omega+t \omega^{\prime}$ is symplectic for each $t \in[0,1]$. Then there is a symplectomorphism $\varphi:(M, \omega) \rightarrow\left(M, \omega^{\prime}\right)$.

The total Liouville volume $V(\omega) \doteq \int_{M} \underbrace{\omega \wedge \cdots \wedge \omega}_{n}$ associated to a symplectic structure $\omega$ on $M$ is a global invariant. That is, if symplectic forms $\omega$ and $\omega^{\prime}$ on a manifold $M$ with $\operatorname{dim}(M)=2 n$ are globally equivalent, their Liouville volumes are equal, $V(\omega)=V\left(\omega^{\prime}\right)$.

In the case of a compact 2-dimensional manifold Moser's theorem implies

Corollary 1. On a compact connected oriented surface $\Sigma$ two symplectic structures $\omega, \omega^{\prime}$ are globally equivalent iff the associated Liouville volumes are equal: $\int_{\Sigma} \omega=\int_{\Sigma} \omega^{\prime}$.

\subsection{The set $\mathcal{G}(\Sigma)$ of topologically stable structures on $\Sigma$.}

Let $\Sigma$ be a compact connected oriented 2-dimensional surface. Since there are no non-trivial 3-vector fields, any bivector field gives rise to a Poisson structure. Thus, Poisson structures on $\Sigma$ form a vector space $\Pi(\Sigma)$.

For $n \geq 0$ let $\mathcal{G}_{n}(\Sigma) \subset \Pi(\Sigma)$ be the set of Poisson structures $\pi$ on $\Sigma$ such that

- the zero set $Z(\pi) \doteq\{p \in \Sigma \mid \pi(p)=0\}$ of $\pi \in \mathcal{G}_{n}(\Sigma)$ consists of $n$ smooth disjoint curves $\gamma_{1}(\pi), \ldots, \gamma_{n}(\pi)$;

- $\pi$ vanishes linearly on each of the curves $\gamma_{1}(\pi), \ldots, \gamma_{n}(\pi)$.

In particular, $\mathcal{G}_{0}(\Sigma)$ is the set of symplectic structures on $\Sigma$. Let $\mathcal{G}(\Sigma) \doteq$ $\bigsqcup_{n \geq 0} \mathcal{G}_{n}(\Sigma)$. The symplectic leaves of a Poisson structure $\pi \in \mathcal{G}(\Sigma)$ are the points in $Z(\pi)=\bigsqcup_{i=1}^{n} \gamma_{i}$ (the 0-dimensional leaves) and the connected components of $\Sigma \backslash Z(\pi)$ (the 2-dimensional leaves.) We call Poisson structures in $\mathcal{G}(\Sigma)$ topologically stable since the topology of their zero sets is unchanged under small perturbations. 
Unless indicated otherwise, throughout the paper we denote by $\omega_{0}$ a symplectic form compatible with the orientation of $\Sigma$ and by $\pi_{0}$ the corresponding Poisson bivector. Since any $\pi$ can be written as $\pi=f \cdot \pi_{0}$ for a function $f \in C^{\infty}(\Sigma)$, we have $\Pi(\Sigma)=C^{\infty}(\Sigma) \cdot \pi_{0}$. The subspace $\mathcal{G}_{n}(\Sigma)$ corresponds in this way to the product $\mathcal{F}_{n}(\Sigma) \cdot \pi_{0}$, where $\mathcal{F}_{n}(\Sigma)$ is the space of smooth functions for which 0 is a regular value and whose zero set consists of $n$ smooth disjoint curves.

According to the Elementary Transversality Theorem (see, e.g., Corollary 4.12 in [3]), the set $\mathcal{F}(\Sigma)=\bigsqcup_{n \geq 0} \mathcal{F}_{n}(\Sigma)$ of functions for which $0 \in \mathbb{R}$ is a regular value forms an open dense subset of $C^{\infty}(\Sigma)$ in the Whitney $C^{\infty}$ topology. Therefore, we have the following

Proposition 1. The set of Poisson structures $\mathcal{G}(\Sigma)$ is generic inside of $\Pi(\Sigma)$, i.e., $\mathcal{G}(\Sigma)$ is an open dense subset of the space $\Pi(\Sigma)$ of all Poisson structures on $\Sigma$ endowed with the Whitney $C^{\infty}$ topology.

Of course, for some sets of disjoint curves on a surface there are no functions (and, therefore, no Poisson structures) vanishing linearly on that set and not zero elsewhere. For example, such is the case of one non-separating curve on a 2 -torus.

We will use the following definition

Definition 2. Two Poisson structures $\pi$ and $\pi^{\prime}$ on an oriented manifold $P$ are globally equivalent if there is an orientation-preserving Poisson isomorphism $\varphi:(P, \pi) \rightarrow\left(P, \pi^{\prime}\right)$.

The main goal of this paper is to classify the set $\mathcal{G}(\Sigma)$ of topologically stable Poisson structures up to global equivalence.

First, we will need the following

Lemma 1. A topologically stable Poisson structure $\pi \in \mathcal{G}_{n}(\Sigma)$ defines an orientation on each of its zero curves $\gamma_{i} \in Z(\pi), i=1, \ldots, n$. Moreover, this induced orientation on the zero curves of $\pi$ does not depend on the choice of orientation of $\Sigma$.

Proof. Let $\omega_{0}$ be a symplectic form on $\Sigma$, and $\pi_{0}=\left(\tilde{\omega}_{0}^{-1} \otimes \tilde{\omega}_{0}^{-1}\right)\left(\omega_{0}\right)$ be the corresponding Poisson bivector. Since $\pi=f \cdot \pi_{0}$ and $f$ vanishes linearly on each of $\gamma_{i} \in Z(\pi)$ and nowhere else, $f$ has constant sign on each of the 2dimensional symplectic leaves of $\pi$. In particular, $f$ has the opposite signs on two leaves having a common bounding curve $\gamma_{i}$. This defines an orientation 
on $\gamma_{i}$ in the following way. For a non-vanishing vector field $X$ tangent to the curve $\gamma_{i}$, we say that $X$ is positive if $\omega_{0}(X, Y) \geq 0$ for all vector fields $Y$ such that $L_{Y} f \geq 0$. We say that $X$ is negative if $-X$ is positive.

Suppose that $X$ is a vector field tangent to $\gamma_{i}$ and positive on $\gamma_{i}$ with respect to the chosen orientation of $\Sigma$. If $\omega_{0}^{\prime}$ is a symplectic form inducing the opposite orientation on $\Sigma$, then $\omega_{0}^{\prime}=-\alpha \cdot \omega_{0}$ with $\alpha \in C^{\infty}(\Sigma), \alpha>0$, and $\pi=-\alpha \cdot f \cdot \pi_{0}$. Since for $Y^{\prime}$ such that $L_{Y^{\prime}}(-\alpha \cdot f) \geq 0$ we have $L_{Y^{\prime}} f \leq 0$, it follows that $\omega_{0}\left(X, Y^{\prime}\right) \leq 0$ and, therefore,

$$
\omega_{0}^{\prime}\left(X, Y^{\prime}\right)=-\alpha \cdot \omega_{0}\left(X, Y^{\prime}\right) \geq 0 .
$$

Hence, if $X$ is positive on $\gamma_{i}$ with respect to a chosen orientation of $\Sigma$, it is also positive on $\gamma_{i}$ with respect to the reverse orientation of $\Sigma$.

We will refer to this orientation of $\gamma_{i} \in Z(\pi)$ as the orientation defined by $\pi$.

\subsection{Diffeomorphism equivalence of sets of disjoint oriented curves.}

We will use the following definition:

Definition 3. Two sets of smooth disjoint oriented curves $\left(\gamma_{1}, \ldots, \gamma_{n}\right)$ and $\left(\gamma_{1}^{\prime}, \ldots, \gamma_{n}^{\prime}\right)$ on an oriented surface $\Sigma$ are called diffeomorphism equivalent (denoted by $\left.\left(\gamma_{1}, \ldots, \gamma_{n}\right) \sim\left(\gamma_{1}, \ldots, \gamma_{n}^{\prime}\right)\right)$ if there is an orientation-preserving diffeomorphism $\varphi: \Sigma \rightarrow \Sigma$ mapping the first set onto the second one and preserving the orientations of curves. That is to say, for each $i \in 1, \ldots, n$, there exists $j \in 1, \ldots, n$, such that $\varphi\left(\gamma_{i}\right)=\gamma_{j}^{\prime}$ (as oriented curves.)

Let $\mathcal{C}_{n}(\Sigma)$ be the space of $n$ disjoint oriented curves on $\Sigma$ and $\mathcal{M}_{n}(\Sigma)$ be the moduli space of $n$ disjoint oriented curves on $\Sigma$ modulo the diffeomorphism equivalence relation, $\mathcal{M}_{n}(\Sigma)=\mathcal{C}_{n}(\Sigma) / \sim$. For a set of disjoint oriented curves $\left(\gamma_{1}, \ldots, \gamma_{n}\right)$, let $\left[\left(\gamma_{1}, \ldots, \gamma_{n}\right)\right] \in \mathcal{M}_{n}(\Sigma)$ denote its class in the moduli space $\mathcal{M}_{n}(\Sigma)$. If $\bigsqcup_{i=1}^{n} \gamma_{i}=Z(\pi)$ for a Poisson structure $\pi$, we will also write $[Z(\pi)] \in \mathcal{M}_{n}$ to denote the class of the set of curves $\left(\gamma_{1}, \ldots, \gamma_{n}\right)$ taken with the orientations defined by $\pi$.

The topology of the inclusion $Z(\pi) \subset \Sigma$ and the orientations of the zero curves of a topologically stable Poisson structure $\pi \in \mathcal{G}(\Sigma)$ are clearly invariant under orientation-preserving Poisson isomorphisms. In other words, if $\pi, \pi^{\prime} \in \mathcal{G}_{n}(\Sigma)$ are globally equivalent, $[Z(\pi)]=\left[Z\left(\pi^{\prime}\right)\right] \in \mathcal{M}_{n}(\Sigma)$. 


\subsection{Modular period invariants.}

Recall the definition and some properties of a modular vector field of a Poisson manifold, introduced by A. Weinstein in [12].

Definition 4 (Weinstein, [12]). For a volume form $\Omega$ on an orientable Poisson manifold $(P, \pi)$ the modular vector field $X^{\Omega}$ of $(P, \pi)$ with respect to $\Omega$ is defined by

$$
X^{\Omega} \cdot h \doteq \frac{L_{X_{h}} \Omega}{\Omega}, \quad h \in C^{\infty}(P)
$$

Here $X_{h}=\tilde{\pi}(d h)$ is the hamiltonian vector field of $h$, where $\tilde{\pi}: T^{*} M \rightarrow T M$ is the canonical bundle map associated to the Poisson bivector $\pi$.

Modular vector fields have the following properties (see [12] for details):

1) $X^{\Omega}=0$ iff $\Omega$ is invariant under the flows of all hamiltonian vector fields of $\pi$;

2) For any other $\Omega^{\prime}$ the difference $X^{\Omega^{\prime}}-X^{\Omega}$ is the hamiltonian vector field $X_{\log \left|\Omega^{\prime} / \Omega\right|}$

3) The flow of $X^{\Omega}$ preserves $\pi$ and $\Omega$, i.e., $L_{X^{\Omega}} \pi=0$ and $L_{X^{\Omega}} \Omega=0$;

4) $X^{\Omega}$ is tangent to the symplectic leaves of maximal dimension.

Let now $\pi \in \mathcal{G}_{n}(\Sigma)$ be a topologically stable Poisson structure on a surface $\Sigma$ as above. A symplectic form $\omega_{0}$ compatible with the orientation of $\Sigma$ is also a volume form on $\Sigma$. Since the modular vector field $X^{\omega_{0}}$ preserves $\pi$, it follows that the restriction of $X^{\omega_{0}}$ to a curve $\gamma_{i} \in Z(\pi)$ is tangent to $\gamma_{i}$ for each $i \in 1, \ldots, n$. Since for another volume form $\omega_{0}^{\prime}$ the difference $X^{\omega_{0}}-X^{\omega_{0}^{\prime}}$ is a hamiltonian vector field and, therefore, vanishes on the zero set of $\pi$, it follows that the restrictions of $X^{\omega_{0}}$ to $\gamma_{1}, \ldots, \gamma_{n}$ are independent of the choice of volume form. It is apparent from the definition of the modular vector field that it is unchanged if the orientation of the surface is reversed.

Suppose that $\pi \in \Pi(\Sigma)$ vanishes linearly on a curve $\gamma$. On a small neighborhood of $\gamma$, let $\theta$ be the coordinate along the flow of the modular vector field $X^{\omega_{0}}$ with respect to $\omega_{0}$ such that $X^{\omega_{0}}=\partial_{\theta}$. Since $\pi$ vanishes linearly on $\gamma$, there exists an annular coordinate neighborhood $(U, z, \theta)$ of 
the curve $\gamma$ such that

$$
\begin{aligned}
& U=\{(z, \theta)|| z \mid<R, \theta \in[0,2 \pi]\}, \quad R>0 \\
& \gamma=\{(z, \theta) \mid z=0\} \\
& \left.\omega_{0}\right|_{U}=d z \wedge d \theta \\
& \left.\pi\right|_{U}=c z \partial_{z} \wedge \partial_{\theta}, \quad c>0
\end{aligned}
$$

Using this coordinates, it is easy to verify the following

Claim 1. The restriction of a modular vector field to a zero curve $\gamma \in Z(\pi)$ on which the Poisson structure vanishes linearly is positive with respect to the orientation on $\gamma$ defined by $\pi$ (see Lemma 1 for the definition of this orientation).

Definition 5 (see also [9]). For a Poisson structure $\pi \in \Pi(\Sigma)$ vanishing linearly on a curve $\gamma \in Z(\pi)$ define the modular period of $\pi$ around $\gamma$ to be

$$
T_{\gamma}(\pi) \doteq \text { period of }\left.X^{\omega_{0}}\right|_{\gamma}
$$

where $X^{\omega_{0}}$ is the modular vector field of $\pi$ with respect to a volume form $\omega_{0}$. Since $\left.X^{\omega_{0}}\right|_{\gamma}$ is independent of choice of $\omega_{0}$, the modular period is welldefined.

Using the coordinate neighborhood $(U, z, \theta)$ of the curve $\gamma$, we obtain

$$
T_{\gamma}(\pi)=\frac{2 \pi}{c}
$$

where $c>0$ is as in (4.)

It turns out that the modular period of the Poisson structure (4) on an annulus $U$ is the only invariant under Poisson isomorphisms:

Lemma 2. Let $U(R)=\{(z, \theta)|| z \mid<R, \theta \in[0,2 \pi]\}$ and $U^{\prime}\left(R^{\prime}\right)=$ $\left\{\left(z^{\prime}, \theta^{\prime}\right)|| z^{\prime} \mid<R^{\prime}, \theta^{\prime} \in[0,2 \pi]\right\}$ be open annuli with the orientations induced by the symplectic forms $\omega_{0}=d z \wedge d \theta$ and $\omega_{0}^{\prime}=d z^{\prime} \wedge d \theta^{\prime}$ respectively. Let $\pi=c z \partial_{z} \wedge \partial_{\theta}, c>0$ and $\pi^{\prime}=c^{\prime} z^{\prime} \partial_{z^{\prime}} \wedge \partial_{\theta^{\prime}}, c^{\prime}>0$ be Poisson structures on $U(R)$ and $U^{\prime}\left(R^{\prime}\right)$ for which the modular periods around the zero curves $\gamma=\{(z, \theta) \mid z=0\}$ and $\gamma^{\prime}=\left\{\left(z^{\prime}, \theta^{\prime}\right) \mid z^{\prime}=0\right\}$ are equal, $T_{\gamma}(\pi)=T_{\gamma^{\prime}}\left(\pi^{\prime}\right)$. Then there is an orientation-preserving Poisson isomorphism $\Phi:(U(R), \pi) \rightarrow\left(U^{\prime}\left(R^{\prime}\right), \pi^{\prime}\right)$. 
Proof. Since the modular periods are equal, we have $c=c^{\prime}$. The map $\Phi:(U(R), \pi) \rightarrow\left(U^{\prime}\left(R^{\prime}\right), \pi^{\prime}\right)$ given by

$$
\Phi(z, \theta)=\left(\frac{R^{\prime}}{R} z, \theta\right)
$$

is a Poisson isomorphism since $\frac{R^{\prime}}{R} z \cdot \frac{R}{R^{\prime}} \partial_{z} \wedge \partial_{\theta}=z \partial_{z} \wedge \partial_{\theta}$. It is easy to see that $\Phi$ preserves the orientation.

The fact that this Poisson isomorphism allows to change the radius of an annuli will be used later in the proof of the classification theorem.

\subsection{The regularized Liouville volume invariant.}

To classify the topologically stable Poisson structures $\mathcal{G}(\Sigma)$ up to orientationpreserving Poisson isomorphisms, we need to introduce one more invariant.

Let $\omega=\left(\tilde{\pi}^{-1} \otimes \tilde{\pi}^{-1}\right)(\pi)$. This form is smooth only on $\Sigma \backslash Z(\pi)$ and defines there a symplectic structure. The symplectic volume of each of the 2-dimensional symplectic leaves is infinite because the form $\omega$ blows up on the curves $\gamma_{1}, \ldots, \gamma_{n} \in Z(\pi)$. However, there is a way to associate a certain finite volume invariant to a Poisson structure in $\mathcal{G}(\Sigma)$, given by the principal value of the integral

$$
V(\pi)=P . V . \int_{\Sigma} \omega .
$$

More precisely, let $h \in C^{\infty}(\Sigma)$ be a function vanishing linearly on $\gamma_{1}, \ldots, \gamma_{n}$ and not zero elsewhere. Let $\mathcal{L}$ be the set of 2 -dimensional symplectic leaves of $\pi$. For $L \in \mathcal{L}$ the boundary $\partial L$ is a union of curves $\gamma_{i_{1}}, \ldots, \gamma_{i_{k}} \in Z(\pi)$. (Note that a leaf $L$ cannot approach the same curve from both sides.) The function $h$ has constant sign on each of the leaves $L \in \mathcal{L}$. Define

$$
V_{h}^{\varepsilon}(\pi) \doteq \int_{|h|>\varepsilon} \omega=\sum_{L \in \mathcal{L}} \int_{L \cap h^{-1}((-\infty,-\varepsilon) \cup(\varepsilon, \infty))} \omega .
$$

Theorem 2. The limit $V(\pi) \doteq \lim _{\varepsilon \rightarrow 0} V_{h}^{\varepsilon}(\pi)$ exists and is independent of the choice of function $h$.

Proof. To show that the limit in the definition of $V(\pi)$ exists and is welldefined, it is enough to argue locally, in a neighborhood of the zero set of the Poisson structure. 
For $i=1, \ldots, n$, let $U_{i}=\left\{\left(z_{i}, \theta_{i}\right)|| z_{i} \mid<R_{i}, \theta_{i} \in[0,2 \pi]\right\}$ be annular coordinate neighborhoods of curves $\gamma_{i}$ such that the restriction of $\pi$ on $U_{i}$ is given by $\pi_{\mid U_{i}}=c_{i} z_{i} \partial_{z_{i}} \wedge \partial_{\theta_{i}}, c_{i}>0$ and $U_{i} \cap Z(\pi)=\gamma_{i}$. Let $\mathcal{U}=\bigsqcup_{i=1}^{n} U_{i}$. Let $h$ be a function vanishing linearly on the curves $\gamma_{1}, \ldots, \gamma_{n}$ and not zero elsewhere. Let $z_{i}^{ \pm}$be functions of $\theta_{i}$ such that $h\left(\theta_{i}, z_{i}^{ \pm}\right)= \pm \varepsilon$. It suffices to show that the limit

$$
\lim _{\varepsilon \rightarrow 0} \mathrm{P} . \mathrm{V} . \int_{0}^{2 \pi} \int_{z_{i}^{-}}^{z_{i}^{+}} \frac{c_{i} d z_{i} \wedge d \theta_{i}}{z_{i}}
$$

is equal to zero. Indeed, this limit is equal to

$$
\int_{0}^{2 \pi}\left(\lim _{\varepsilon \rightarrow 0} \mathrm{P} . \mathrm{V} . \int_{z_{i}^{-}}^{z_{i}^{+}} \frac{c_{i} d z_{i}}{z_{i}}\right) d \theta_{i}=c_{i} \int_{0}^{2 \pi} \lim _{\varepsilon \rightarrow 0} \ln \left|\frac{z_{i}^{+}}{z_{i}^{-}}\right| d \theta_{i}=0 .
$$

Hence $V(\pi) \in \mathbb{R}$ is a global equivalence invariant of a Poisson structure $\pi \in \mathcal{G}(\Sigma)$ on an oriented surface which we call the regularized Liouville volume since in the case of a symplectic structure (i.e., $\pi \in \mathcal{G}_{0}(\Sigma)$ ) it is exactly the Liouville volume. If we reverse the orientation of $\Sigma$, the regularized volume invariant changes sign.

\section{A classification of topologically stable Poisson structures.}

Theorem 3. Topologically stable Poisson structures $\mathcal{G}_{n}(\Sigma)$ on a compact connected oriented surface $\Sigma$ are completely classified (up to an orientationpreserving Poisson isomorphism) by the following data:

1) The equivalence class $[Z(\pi)] \in \mathcal{M}_{n}(\Sigma)$ of the set $Z(\pi)=\bigsqcup_{i=1}^{n} \gamma_{i}$ of zero curves with orientations defined by $\pi$;

2) The modular periods around the zero curves $\left\{\gamma_{i} \mapsto T_{\gamma_{i}}(\pi) \mid i=\right.$ $1, \ldots, n\}$;

3) The regularized Liouville volume $V(\pi)$.

In other words, two Poisson structures $\pi, \pi^{\prime} \in \mathcal{G}_{n}(\Sigma)$ are globally equivalent if and only if their sets of oriented zero curves are diffeomorphism equivalent, the modular periods around the corresponding curves are the same, and the regularized Liouville volumes are equal.

To prove this result we will need the following 
Lemma 3. Let $D$ be a connected 2-dimensional manifold, and $\omega_{1}, \omega_{2}$ be two symplectic forms on $D$ inducing the same orientation and such that

- $\left.\omega_{1}\right|_{D \backslash K}=\left.\omega_{2}\right|_{D \backslash K}$ for a compact set $K \subset D$;

- $\int_{D} \omega_{1}=\int_{D} \omega_{2}$;

Then there exists a symplectomorphism $\varphi:\left(D, \omega_{1}\right) \rightarrow\left(D, \omega_{2}\right)$ such that $\left.\varphi\right|_{D \backslash K}=i d$.

Proof. (Moser's trick.) Let $\omega_{t} \doteq \omega_{1} \cdot(1-t)+\omega_{2} \cdot t$ for $t \in[0,1]$. Since $\left.\omega_{1}\right|_{D \backslash K}=$ $\left.\omega_{2}\right|_{D \backslash K}$, the form $\Delta(\omega) \doteq \omega_{2}-\omega_{1}$ is compactly supported $(\operatorname{supp}(\Delta(\omega)) \subseteq K$.) Since $\int_{D} \omega_{1}=\int_{D} \omega_{2}$, the class of $\Delta(\omega)$ in the second de Rham cohomology with compact support $H_{\mathrm{c}}^{2}(D)$ is trivial. Hence $\Delta(\omega)=d \mu$ for a 1 -form $\mu \in \Omega_{\mathrm{c}}^{1}(D)$. Then for $v_{t} \doteq-\tilde{\omega}_{t}^{-1}(\mu)$ we have

$$
L_{v_{t}} \omega_{t}=d v_{v_{t}} \omega_{t}+\imath_{v_{t}} d \omega_{t}=d \imath_{v_{t}} \omega_{t}=-\Delta(\omega) \text {. }
$$

Therefore,

$$
L_{v_{t}} \omega_{t}+\frac{d \omega_{t}}{d t}=0
$$

Let $\rho_{t}$ be the flow of the time-dependent vector field $v_{t}$. Since

$$
\frac{d}{d t}\left(\rho_{t}^{*} \omega_{t}\right)=\rho_{t}^{*}\left(L_{v_{t}} \omega_{t}+\frac{d \omega_{t}}{d t}\right)=0
$$

$\rho_{t}^{*} \omega_{t}=\omega_{1}$ for all $t \in[0,1]$. Since $v_{t}=0$ outside of $K$, it follows that $\left.\rho_{t}\right|_{D \backslash K}=$ id. Define $\varphi=\rho_{1}$. Then $\varphi_{\mid D \backslash K}=$ id and $\varphi^{*} \omega_{2}=\rho_{1}^{*} \omega_{2}=\omega_{1}$ as desired.

We now have all of the ingredients for the proof of Theorem 3 .

Proof of Theorem 3. Let $\pi$ and $\pi^{\prime}$ be two topologically stable Poisson structures. Clearly, the coincidence of all of the invariants listed in the theorem is necessary for $\pi$ and $\pi^{\prime}$ to be isomorphic.

Conversely, assume that all the invariants are the same. Then the zero sets $Z(\pi) \subset \Sigma$ and $Z\left(\pi^{\prime}\right) \subset \Sigma$ are diffeomorphic and, therefore, we may assume that $Z(\pi)=Z\left(\pi^{\prime}\right)$ by replacing $\pi^{\prime}$ with an isomorphic structure. Since both $\pi=f \cdot \pi_{0}$ and $\pi^{\prime}=f^{\prime} \cdot \pi_{0}$ vanish linearly on each connected component $\gamma \subset Z(\pi)=Z\left(\pi^{\prime}\right)$, we can, by once again replacing $\pi^{\prime}$ by a 
Poisson-isomorphic structure, assume that in an annular coordinate neighborhood $U_{i} \simeq S^{1} \times(-1,1)=\left\{\left(z_{i}, \theta_{i}\right)\left|\theta_{i} \in[0,2 \pi],\right| z_{i} \mid<R_{i}\right\}$ of $\gamma_{i}$ we have

$$
\begin{aligned}
\left.\pi\right|_{U_{i}} & =c_{i} z_{i} \partial_{z_{i}} \wedge \partial_{\theta}, \quad c_{i} \neq 0, \\
\left.\pi^{\prime}\right|_{U_{i}} & =c_{i}^{\prime} z_{i} \partial_{z_{i}} \wedge \partial_{\theta}, \quad c_{i}^{\prime} \neq 0 .
\end{aligned}
$$

Since the modular periods of $\pi$ and $\pi^{\prime}$ around the corresponding curves are assumed to be the same, we have $2 \pi /\left|c_{i}\right|=2 \pi /\left|c_{i}^{\prime}\right|$, which implies $\left|c_{i}\right|=\left|c_{i}^{\prime}\right|$. The fact that the orientations of the connected components of $Z(\pi)$ and $Z\left(\pi^{\prime}\right)$ induced by modular vector fields are the same implies that $c_{i}=c_{i}^{\prime}$. Hence $\pi$ and $\pi^{\prime}$ are equal in a neighborhood of $Z(\pi)$. By replacing $\pi^{\prime}$ with an isomorphic structure we may assume that $\pi=\pi^{\prime}$ on a neighborhood $U$ of $Z(\pi)$.

Consider the non-compact manifold $N \doteq \Sigma \backslash Z(\pi)$. The desired isomorphism of $\pi$ and $\pi^{\prime}$ on $\Sigma$ would follow if we could argue that $\pi$ and $\pi^{\prime}$ are isomorphic on $N$ via a diffeomorphism $\varphi$ which is an identity on $N \cap U$. Indeed, $\varphi$ could then be extended to a diffeomorphism $\bar{\varphi}$ of $\Sigma$ by setting $\varphi(p)=p$ for $p \in Z(\pi)$ and we would clearly have $\bar{\varphi}_{*}(\pi)=\pi^{\prime}$. Since $\pi$ and $\pi^{\prime}$ are nonzero on $N$, they come from symplectic structures $\frac{\omega_{0}}{f}$ and $\frac{\omega_{0}}{f^{\prime}}$ on $N$. These 2 -forms coincide on $N \cap U$. Moreover, $\int_{N}\left(\frac{\omega_{0}}{f}-\frac{\omega_{0}}{f^{\prime}}\right)=V(\pi)-V\left(\pi^{\prime}\right)=0$. Hence $\frac{\omega_{0}}{f}$ and $\frac{\omega_{0}}{f^{\prime}}$ define the same class in the compactly-supported de Rham cohomology of $N$. It is now a matter of imitating the proof of Moser's theorem to construct a time-dependent vector field supported on $N \backslash U$ and whose flow at time 1 carries $\frac{\omega_{0}}{f}$ into $\frac{\omega_{0}}{f^{\prime}}$. Taking $\varphi$ to be that flow at time 1 we find that we have obtained the desired isomorphism.

Remark 1. Given $\pi, \pi^{\prime} \in \mathcal{S}_{n}(\Sigma)$, one can ask if $(\Sigma, \pi)$ and $\left(\Sigma, \pi^{\prime}\right)$ are equivalent by an arbitrary (possibly orientation-reversing) Poisson isomorphism. Fix an orientation-reversing diffeomorphism $\nu: \Sigma \rightarrow \Sigma$. Then $\pi$ and $\pi^{\prime}$ are equivalent by an orientation-reversing diffeomorphism if and only if $\nu_{*} \pi$ and $\pi^{\prime}$ are equivalent by an orientation-preserving diffeomorphism. It is not hard to see that $T_{\gamma_{i}}(\pi)=T_{\nu\left(\gamma_{i}\right)}\left(\nu_{*} \pi\right)$ for all $\gamma_{i} \in Z(\pi)$ and $V(\pi)=-V\left(\nu_{*} \pi\right)$. Thus the question of equivalence by orientation reversing maps can be reduced to the orientation-preserving context of Theorem 3 .

Example 1. Let $\omega$ and $\omega^{\prime}=-\omega$ be two symplectic structures on a compact oriented surface. Then $\omega$ and $\omega^{\prime}$ are Poisson isomorphic by an orientationreversing diffeomorphism, but not by an orientation-preserving diffeomorphism.

There are, of course, similar examples of structures with non-trivial sets of linear degeneracy. Consider the unit 2-sphere $S^{2}$ with the cylindrical polar 
coordinates $(z, \theta)$ away from its poles. Let $\omega_{0}=d z \wedge d \theta$ be a symplectic form on $S^{2}$ with the corresponding Poisson bivector $\pi_{0}$. Let $\pi, \pi^{\prime} \in \mathcal{G}_{2}\left(S^{2}\right)$ be the Poisson structures given by

$$
\pi=(z-a)(z-b) \partial_{z} \wedge \partial_{\theta}, \quad-1<b<a<1
$$

and $\pi^{\prime}=-\pi$. Choose $a$ and $b$ in such a way that $V(\pi)=V\left(\pi^{\prime}\right)=0$. Let $\gamma_{1}=\{(z, \theta) \mid z=a\}$ and $\gamma_{2}=\{(z, \theta) \mid z=b\}$ be the zero curves of $\pi, \pi^{\prime}$. On both $\gamma_{1}$ and $\gamma_{2}$ the orientations defined by $\pi$ and $\pi^{\prime}$ are opposite to each other. Let $L_{\text {top }}=\{(z, \theta) \mid a<z<1\}, L_{\text {middle }}=\{(z, \theta) \mid b<z<a\}$ and $L_{\text {bottom }}=\{(z, \theta) \mid-1<z<b\}$ be the 2-dimensional leaves (common for both structures). The structures $\pi$ and $\pi^{\prime}$ can not be Poisson isomorphic in an orientation-preserving way since such a diffeomorphism would have to exchange the two-dimensional disks $L_{\text {top }}$ and $L_{\text {bottom }}$ with the annulus $L_{\text {middle }}$. On the other hand, $\left(S^{2}, \pi\right)$ and $\left(S^{2}, \pi^{\prime}\right)$ are clearly Poisson isomorphic by an orientation-reversing diffeomorphism $(z, \theta) \mapsto(z,-\theta)$.

\section{Poisson cohomology of topologically stable structures.}

In this section we compute the Poisson cohomology of a given topologically stable Poisson structure on a compact connected oriented surface and describe its relation to the infinitesimal deformations and the classifying invariants introduced above. (For generalities on Poisson cohomology see, e.g., [10].)

First, recall the following

Lemma 4 (e.g., Roytenberg [9]). The Poisson cohomology of an annular neighborhood $U=\{(z, \theta)|| z \mid<R, \theta \in[0,2 \pi]\}$ of a zero curve $\gamma$ on which $\pi_{\mid U}=z \partial_{z} \wedge \partial_{\theta}$ is given by

$$
\begin{aligned}
& H_{\pi}^{0}\left(U, \pi_{\mid U}\right)=\operatorname{span}\langle 1\rangle=\mathbb{R}, \\
& H_{\pi}^{1}\left(U, \pi_{\mid U}\right)=\operatorname{span}\left\langle\partial_{\theta}, z \partial_{z}\right\rangle=\mathbb{R}^{2}, \\
& H_{\pi}^{2}\left(U, \pi_{\mid U}\right)=\operatorname{span}\left\langle z \partial_{z} \wedge \partial_{\theta}\right\rangle=\mathbb{R} .
\end{aligned}
$$

Thus, $H_{\pi}^{0}(U)$ is generated by constant functions. The first cohomology $H_{\pi}^{1}(U)$ is generated by the modular class $\left(\partial_{\theta}\right.$ is the modular vector field of $\pi_{\mid U}$ with respect to $\omega_{0}=d z \wedge d \theta$ ) and the image of the first de Rham cohomology class of $U$ (spanned by $d \theta$ ) under the homomorphism $\tilde{\pi}: H^{1}(U) \rightarrow H_{\pi}^{1}(U)$, which is injective in this case. The second cohomology is generated by $\pi_{\mid U}$.

Let $\pi \in \mathcal{G}_{n}(\Sigma)$ be a topologically stable Poisson structure on $\Sigma$. Since a Casimir function on $\Sigma$ must be constant on all connected components of 
$\Sigma \backslash Z(\pi)$, by continuity it must be constant everywhere. Hence $H_{\pi}^{0}(\Sigma)=$ $\mathbb{R}=\operatorname{span}\langle 1\rangle$.

We will (inductively) use the Mayer-Vietoris sequence of Poisson cohomology (see, e.g., [10]) to compute $H_{\pi}^{1}(\Sigma)$ and $H_{\pi}^{2}(\Sigma)$.

Let $U_{i}$ be an annular neighborhood of the curve $\gamma_{i} \in Z(\pi)$ such that $U_{i} \cap Z(\pi)=\gamma_{i}$. Let $V_{0} \doteq \Sigma$ and define inductively $V_{i} \doteq V_{i-1} \backslash \gamma_{i}$ for $i=1, \ldots, n$. Consider the cover of $V_{i-1}$ by open sets $U_{i}$ and $V_{i}$. Consider the exact Mayer-Vietoris sequence of Poisson cohomology associated to this cover:

$$
\begin{aligned}
& 0 \rightarrow H_{\pi}^{0}\left(V_{i-1}\right) \stackrel{\alpha_{i}^{0}}{\rightarrow} H_{\pi}^{0}\left(U_{i}\right) \oplus H_{\pi}^{0}\left(V_{i}\right) \stackrel{\beta_{i}^{0}}{\rightarrow} H_{\pi}^{0}\left(U_{i} \cap V_{i}\right) \quad \stackrel{\delta_{i}^{0}}{\rightarrow} \\
& \rightarrow H_{\pi}^{1}\left(V_{i-1}\right) \stackrel{\alpha_{i}^{1}}{\rightarrow} H_{\pi}^{1}\left(U_{i}\right) \oplus H_{\pi}^{1}\left(V_{i}\right) \stackrel{\beta_{i}^{1}}{\rightarrow} H_{\pi}^{1}\left(U_{i} \cap V_{i}\right) \quad \stackrel{\delta_{i}^{1}}{\rightarrow} \\
& \rightarrow H_{\pi}^{2}\left(V_{i-1}\right) \stackrel{\alpha_{i}^{2}}{\rightarrow} H_{\pi}^{2}\left(U_{i}\right) \oplus H_{\pi}^{2}\left(V_{i}\right) \stackrel{\beta_{i}^{2}}{\rightarrow} H_{\pi}^{2}\left(U_{i} \cap V_{i}\right) \quad \rightarrow 0 .
\end{aligned}
$$

By exactness, $H_{\pi}^{1}\left(V_{i-1}\right) \simeq \operatorname{Im}\left(\delta_{i}^{0}\right) \oplus \operatorname{ker} \beta_{i}^{1}$, where

$$
\beta_{i}^{1}\left([\chi]_{U_{i}},[\nu]_{V_{i}}\right)=[\chi-\nu]_{U_{i} \cap V_{i}}, \quad \chi \in X_{\pi}^{1}\left(U_{i}\right), \nu \in X_{\pi}^{1}\left(V_{i}\right), d_{\pi} \chi=d_{\pi} \nu=0,
$$

and $[X]_{W}$ denotes the class of the (Poisson) vector field $\left.X\right|_{W}$ in $H_{\pi}^{1}(W)$.

By Lemma $4, H_{\pi}^{1}\left(U_{i}\right) \simeq \tilde{\pi}\left(H^{1}\left(U_{i}\right)\right) \oplus \operatorname{span}\left\langle\partial_{\theta_{i}}\right\rangle \simeq \mathbb{R}^{2}$. Since $U_{i} \cap V_{i}$ is a union of two symplectic annuli, $H_{\pi}^{1}\left(U_{i} \cap V_{i}\right)=\tilde{\pi}\left(H^{1}\left(U_{i} \cap V_{i}\right)\right)=\mathbb{R}^{2}$. Therefore,

$$
H_{\pi}^{1}\left(V_{i-1}\right) \simeq \delta_{i}^{0}\left(H_{\pi}^{0}\left(U_{i} \cap V_{i}\right)\right) \oplus \operatorname{span}\left\langle\partial_{\theta_{i}}\right\rangle \oplus \operatorname{ker}\left(\left.\beta_{i}^{1}\right|_{\tilde{\pi}\left(H^{1}\left(U_{i}\right)\right) \oplus H_{\pi}^{1}\left(V_{i}\right)}\right) .
$$

Consider also the long exact sequence in de Rham cohomology associated to the same cover

$$
\begin{array}{rllllll}
0 & \rightarrow H^{0}\left(V_{i-1}\right) & \stackrel{a_{i}^{0}}{\rightarrow} & H^{0}\left(U_{i}\right) \oplus H^{0}\left(V_{i}\right) & \stackrel{b_{i}^{0}}{\rightarrow} & H^{0}\left(U_{i} \cap V_{i}\right) & \stackrel{d_{\dot{m}}^{0}}{\rightarrow} \\
& \rightarrow H^{1}\left(V_{i-1}\right) & \stackrel{a_{\dot{1}}^{1}}{\rightarrow} & H^{1}\left(U_{i}\right) \oplus H^{1}\left(V_{i}\right) & \stackrel{b_{i}^{1}}{\rightarrow} & H^{1}\left(U_{i} \cap V_{i}\right) & \stackrel{d_{\dot{1}}}{\rightarrow} \\
& \rightarrow H^{2}\left(V_{i-1}\right) & \stackrel{a_{i}^{2}}{\rightarrow} & H^{2}\left(U_{i}\right) \oplus H^{2}\left(V_{i}\right) \stackrel{b_{i}^{2}}{\rightarrow} & H^{2}\left(U_{i} \cap V_{i}\right) & \rightarrow 0 .
\end{array}
$$

By exactness, we have $H^{1}\left(V_{i-1}\right) \simeq \operatorname{Im}\left(d_{i}^{0}\right) \oplus \operatorname{ker} b_{i}^{1}$. Since $V_{n}=\Sigma \backslash Z(\pi)$ is symplectic, $H_{\pi}^{*}\left(V_{n}\right)=\tilde{\pi}\left(H^{*}\left(V_{n}\right)\right)$. This together with $H_{\pi}^{0}\left(U_{n}\right) \simeq H^{0}\left(U_{n}\right)$, $H_{\pi}^{0}\left(U_{n} \cap V_{n}\right)=\tilde{\pi}\left(H^{0}\left(U_{n} \cap V_{n}\right)\right)$ implies $\operatorname{Im}\left(\delta_{n}^{0}\right)=\tilde{\pi}\left(\operatorname{Im}\left(d_{n}^{0}\right)\right)$ and, therefore,

$$
\operatorname{ker}\left(\left.\beta_{n}^{1}\right|_{\tilde{\pi}\left(H^{1}\left(U_{n}\right) \oplus H_{\pi}^{1}\left(V_{n}\right)\right)}\right)=\tilde{\pi}\left(\operatorname{ker}\left(b_{n}^{1}\right)\right) .
$$

Hence, from (7) it follows that

$$
H_{\pi}^{1}\left(V_{n-1}\right) \simeq \tilde{\pi}\left(\operatorname{Im}\left(d_{n-1}\right)\right) \oplus \operatorname{span}\left\langle\partial_{\theta_{n}}\right\rangle \oplus \tilde{\pi}\left(H^{1}\left(V_{n-1}\right)\right) .
$$


For $i=n-2$, we have

$$
\begin{aligned}
H_{\pi}^{1}\left(V_{n-2}\right) & \simeq \operatorname{span}\left\langle\partial_{\theta_{n-1}}\right\rangle \oplus \operatorname{ker}\left(\left.\beta_{n-1}^{1}\right|_{\tilde{\pi}\left(H^{1}\left(U_{n-1}\right) \oplus\left(\tilde{\pi}\left(H^{1}\left(V_{n-1}\right)\right) \oplus \tilde{\pi}\left(\operatorname{Im}\left(d_{i}^{0}\right)\right)\right)\right.}\right) \\
& =\operatorname{span}\left\langle\partial_{\theta_{n-1}}\right\rangle \oplus \operatorname{span}\left\langle\partial_{\theta_{n}}\right\rangle \oplus \tilde{\pi}\left(H^{1}\left(V_{n-2}\right)\right) .
\end{aligned}
$$

Working inductively (from $i=n-1$ to $i=0$ ), we obtain

$$
H_{\pi}^{1}(\Sigma) \simeq \mathbb{R}^{2 g+n}=H^{1}(\Sigma) \oplus \bigoplus_{i=1}^{n} \operatorname{span}\left\langle\partial_{\theta_{i}}\right\rangle,
$$

where $g$ is the genus of the surface $\Sigma$.

For the second Poisson cohomology, we have $H_{\pi}^{2}\left(U_{i} \cap V_{i}\right)=0, H_{\pi}^{2}\left(U_{i}\right)=\mathbb{R}$ for all $i=1, \ldots, n$ and $H_{\pi}^{2}\left(V_{n}\right)=0$. Therefore, applying the Mayer-Vietoris sequence inductively, we obtain

$$
H_{\pi}^{2}\left(V_{i-1}\right) \simeq \mathbb{R} \oplus H_{\pi}^{2}\left(U_{i}\right) \oplus H_{\pi}^{2}\left(V_{i}\right),
$$

where $\mathbb{R}$ is generated by the image of $\left(r_{i} \partial_{r_{i}},-r_{i} \partial_{r_{i}}\right) \in H_{\pi}^{1}\left(U_{i} \cap V_{i}\right)$ under the connecting homomorphism $\delta_{i}^{0}$. One can show (similarly to Lemma 4.3.1 in [9]) that the class of the standard non-degenerate Poisson structure $\left.\pi_{0}\right|_{V_{i-1}}$ is not trivial in $H_{\pi}^{2}\left(V_{i-1}\right)$. Therefore,

$$
H_{\pi}^{2}(\Sigma) \simeq \mathbb{R}^{n+1}=\bigoplus_{i=1}^{n} H_{\pi}^{2}\left(U_{i}\right) \oplus \mathbb{R},
$$

where the first $n$ generators are the Poisson structures of the form

$$
\pi_{i} \doteq \pi \cdot\left\{\text { bump function around the curve } \gamma_{i}\right\}, \quad i=1, \ldots, n,
$$

and the last generator is the standard non-degenerate Poisson structure $\pi_{0}$ on $\Sigma$. Therefore, we have proved the following

Theorem 4. Let $\pi \in \mathcal{G}_{n}(\Sigma)$ be a topologically stable Poisson structure on a compact connected oriented surface $\Sigma$ of genus $g$. The Poisson cohomology of $\pi$ is given by

$$
\begin{aligned}
& H_{\pi}^{0}(\Sigma, \pi)=\operatorname{span}\langle 1\rangle=\mathbb{R} \\
& H_{\pi}^{1}(\Sigma, \pi)=\operatorname{span}\left\langle X^{\omega_{0}}\left(\pi_{1}\right), \ldots, X^{\omega_{0}}\left(\pi_{n}\right)\right\rangle \oplus \tilde{\pi}\left(H_{\mathrm{deRham}}^{1}(\Sigma)\right)=\mathbb{R}^{n+2 g}, \\
& H_{\pi}^{2}(\Sigma, \pi)=\operatorname{span}\left\langle\pi_{0} ; \pi_{1}, \ldots, \pi_{n}\right\rangle=\mathbb{R}^{n+1},
\end{aligned}
$$

where $\pi_{0}$ is a non-degenerate Poisson structure on $\Sigma, \pi_{i} \in \mathcal{G}_{1}(\Sigma), i=$ $1, \ldots, n$ is a Poisson structure vanishing linearly on $\gamma_{i} \in Z(\pi)$ and identically zero outside of a neighborhood of $\gamma_{i}$; and $X^{\omega_{0}}\left(\pi_{i}\right)$ is the modular vector field of $\pi_{i}$ with respect to the standard symplectic form $\omega_{0}$ on $\Sigma$. 
Notice that the dimensions of the cohomology spaces depend only on the number of the zero curves and not on their positions. In particular, the Poisson cohomology as a vector space does not depend on the homology classes of the zero curves of the structure. Recall (see, e.g., [10]) that the Poisson cohomology space $H_{\pi}^{*}(P)$ has the structure of an associative graded commutative algebra induced by the operation of wedge multiplication of multivector fields. A direct computation verifies the following

Proposition 2. The wedge product on the cohomology space $H_{\pi}^{*}(\Sigma, \pi)$ of a topologically stable Poisson structure on $\Sigma$ is determined by

$$
\begin{aligned}
& {[1] \wedge[\chi]=[\chi], \quad[\chi] \in H_{\pi}^{*}(\Sigma, \pi),} \\
& {\left[X^{\omega_{0}}\left(\pi_{i}\right)\right] \wedge\left[X^{\omega_{0}}\left(\pi_{j}\right)\right]=0, \quad i, j=1, \ldots, n} \\
& {\left[X^{\omega_{0}}\left(\pi_{i}\right)\right] \wedge[\tilde{\pi}(\alpha)]=\left[\alpha\left(X^{\omega_{0}}\left(\pi_{i}\right)\right) \cdot \pi_{i}\right]=\left(\frac{1}{T_{\gamma_{i}}(\pi)} \int_{\gamma_{i}} \alpha\right) \cdot\left[\pi_{i}\right],} \\
& {[\tilde{\pi}(\alpha)] \wedge\left[\tilde{\pi}\left(\alpha^{\prime}\right)\right]=\tilde{\pi}\left(\bar{\alpha} \wedge \overline{\alpha^{\prime}}\right)} \\
& {[\chi] \wedge H_{\pi}^{2}(\Sigma, \pi)=0, \quad[\chi] \in H_{\pi}^{1}(\Sigma) \oplus H_{\pi}^{2}(\Sigma)}
\end{aligned}
$$

where $\alpha, \alpha^{\prime} \in \Omega^{1}(\Sigma), d \alpha=d \alpha^{\prime}=0$.

(Here bar denotes the class of its argument in the de Rham cohomology and the brackets [ ] denote the class in the Poisson cohomology.) We should mention that the wedge product $\wedge$ in de Rham cohomology is dual to the intersection product in homology.

This result allows one to compute the number of zero curves $\gamma_{k}$, which determine non-zero homology classes. To see this, we note that $\left[\pi_{k}\right] \in$ $H_{\pi}^{1}(\Sigma) \wedge H_{\pi}^{1}(\Sigma)$ iff there exists a 1 -form $\alpha$ such that $\int_{\gamma_{k}} \alpha \neq 0$, i.e., $\gamma_{k}$ is nonzero in homology. If $\Sigma$ is not a sphere, $H_{\text {deRham }}^{1}(\Sigma)$ is non-zero. Since the intersection form on $H_{\mathrm{deRham}}^{1}(\Sigma)$ is non-degenerate (implementing Poincare duality), it follows that $H_{\text {deRham }}^{1}(\Sigma) \wedge H_{\text {deRham }}^{1}(\Sigma) \neq 0$. Thus in the case that $\Sigma$ is not a sphere, $H_{\pi}^{1}(\Sigma) \wedge H_{\pi}^{1}(\Sigma)$ has the set

$$
\left\{\pi_{0}\right\} \cup\left\{\pi_{k}: \gamma_{k} \text { is nontrivial in homology }\right\}
$$

as a basis and so the number of curves $\gamma_{k}$, which are non-trivial in homology, is just $\operatorname{dim}\left(H_{\pi}^{1}(\Sigma) \wedge H_{\pi}^{1}(\Sigma)\right)-1$. In the case that $\Sigma$ is a sphere, all $\gamma_{k}$ are of course topologically trivial.

We can now interpret the generators of $H_{\pi}^{2}(\Sigma, \pi)$ as infinitesimal deformations of the Poisson structure $\pi$ and find out how these deformations affect the classifying invariants. 
Corollary 2. Let $\pi \in \mathcal{G}_{n}(\Sigma), Z(\pi)=\bigsqcup_{i=1}^{n} \gamma_{i}$. The following $n+1$ oneparameter families of infinitesimal deformations form a basis of $H_{\pi}^{2}(\Sigma, \pi)$

(1) $\pi \mapsto \pi+\varepsilon \cdot \pi_{0}$;

(2) $\pi \mapsto \pi+\varepsilon \cdot \pi_{i}, i=1, \ldots, n$.

Each of these deformations changes exactly one of the classifying invariants of the Poisson structure: $\pi \mapsto \pi+\varepsilon \cdot \pi_{0}$ changes the regularized Liouville volume and $\pi \mapsto \pi+\varepsilon \cdot \pi_{i}$ changes the modular period around the curve $\gamma_{i}$ for each $i=1, \ldots, n$.

\section{Example: topologically stable Poisson structures on the sphere.}

It would be interesting to describe the moduli space of the space of topologically stable Poisson structures on an oriented surface up to orientationpreserving diffeomorphisms. The first step would be the description of the moduli space $\mathcal{M}_{n}$ of $n$ disjoint oriented curves on $\Sigma$. However, this problem is already quite difficult for a general surface. Here we will consider the simplest example of topologically stable Poisson structures on the sphere.

Let $\left(\gamma_{1}, \ldots, \gamma_{n}\right)$ be a set of disjoint curves on $S^{2}$. Let $\mathcal{S}_{1}, \ldots, \mathcal{S}_{n+1}$ be the connected components of $S^{2} \backslash\left(\gamma_{1}, \ldots, \gamma_{n}\right)$. To the configuration of curves $\left(\gamma_{1}, \ldots, \gamma_{n}\right)$ we associate a graph $\Gamma\left(\gamma_{1}, \ldots, \gamma_{n}\right)$ in the following way. The vertices $v_{1}, \ldots, v_{n+1}$ of the graph correspond to the connected components $\mathcal{S}_{1}, \ldots, \mathcal{S}_{n+1}$. Two vertices $v_{i}$ and $v_{j}$ are connected by an edge $e_{k}$ iff $\gamma_{k}$ is the common bounding curve of the regions $\mathcal{S}_{i}$ and $\mathcal{S}_{j}$.

Claim 2. For a set of disjoint curves $\gamma_{1}, \ldots, \gamma_{n}$ on $S^{2}$ the graph $\Gamma\left(\gamma_{1}, \ldots\right.$, $\left.\gamma_{n}\right)$ is a tree.

Proof. Let $e_{i} \in E\left(\Gamma\left(\gamma_{1}, \ldots, \gamma_{n}\right)\right)$ be an edge of the graph corresponding to the curve $\gamma_{i}$. Since $S^{2} \backslash \gamma_{i}$ is a union of two open sets, it follows that $\Gamma\left(\gamma_{1}, \ldots, \gamma_{n}\right) \backslash e_{i}$ (i.e., the graph $\Gamma\left(\gamma_{1}, \ldots, \gamma_{n}\right)$ with the edge $e_{i}$ removed) is a union of two disjoint graphs. Since this is true for any $e_{i}, i=1, \ldots, n$, the graph is a tree.

Choose an orientation on $S^{2}$ and a symplectic form $\omega_{0}$ (with the Poisson bivector $\pi_{0}$ ) which induces this orientation. Let $\pi \in \mathcal{G}_{n}(\Sigma)$ be a topologically stable Poisson structure. The function $f=\pi / \pi_{0}$ has constant signs on the 
2-dimensional symplectic leaves. Let $\Gamma\left(\gamma_{1}, \ldots, \gamma_{n}\right)$ be the tree associated to the zero curves $\gamma_{1}, \ldots, \gamma_{n}$ of $\pi$ as described above. Assign to each vertex $v_{i}$ a sign (plus or minus) equal to the sign of the function $f$ on the corresponding symplectic leaf $\mathcal{S}_{i}$ of $\pi$. The properties of $\pi$ imply that for any edge of this tree its ends are assigned the opposite signs. We will call the tree associated to the zero curves of $\pi$ with signs associated to its vertices the signed tree $\Gamma(\pi)$ of the Poisson structure $\pi$.

Consider the map $T_{e}(\pi): E(\Gamma(\pi)) \rightarrow \mathbb{R}^{+}$which for each edge $e \in$ $E(\Gamma(\pi))$ gives a period $T_{e}(\pi)$ of a modular vector field of $\pi$ around the zero curve corresponding to this edge. The classification Theorem 3 implies

Theorem 5. The topologically stable Poisson structures $\pi \in \mathcal{G}_{n}\left(S^{2}\right)$ on the sphere are completely classified (up to an orientation-preserving Poisson isomorphism) by the signed tree $\Gamma(\pi)$, the map $e \mapsto T_{e}(\pi)$, e $\in E(\Gamma(\pi))$ and the regularized Liouville volume $V(\pi)$. In other words, $\pi_{1}, \pi_{2} \in \mathcal{G}_{n}\left(S^{2}\right)$ are globally equivalent if and only if the corresponding $\Gamma\left(\pi_{i}\right),\left\{e \mapsto T_{e}\left(\pi_{i}\right)\right\}$, $V\left(\pi_{i}\right)$ are the same (up to automorphisms of signed trees with positive numbers attached to their edges).

The moduli space of generic Poisson structures in $\mathcal{G}_{n}\left(S^{2}\right)$ up to Poisson isomorphisms is

$$
\mathcal{G}_{n}\left(S^{2}\right) /(\text { Poisson isomorphisms }) \simeq\left(\bigsqcup_{\Gamma_{n+1}}\left(\mathbb{R}^{+}\right)^{n} / \operatorname{Aut}\left(\Gamma, T_{e}\right)\right) \times \mathbb{R},
$$

where $\operatorname{Aut}\left(\Gamma, T_{e}\right)$ is the automorphism group of the signed tree $\Gamma$ with $n+1$ vertices and with positive numbers $T_{e}$ attached to its edges $e \in E(\Gamma(\pi))$. The moduli space has dimension $n+1$ and is coordinatized by $\left\{T_{e}(\pi) \mid e \in\right.$ $E(\Gamma(\pi))\}$ and $V(\pi)$.

A particular case of topologically stable Poisson structures on $S^{2}$, the $S U(2)$-covariant structures vanishing on a circle on $S^{2}$, were considered by D.Roytenberg in [9]. In the coordinates $(z, \theta)$ on the unit sphere these structures are given by

$$
\pi_{b}=a(z-b) \partial_{z} \wedge \partial_{\theta} \quad \text { for }|b|<1, a>0 .
$$

The modular period around the zero curve (a "horizontal" circle $\gamma=$ $\{(z, \theta) \mid z=b\})$ and the regularized Liouville volume are given by

$$
\begin{aligned}
& T_{\gamma=\{(z, \theta) \mid z=b\}}(\pi)=\frac{2 \pi}{a}, \\
& V(\pi)=\frac{2 \pi}{a} \ln \frac{1+b}{1-b} .
\end{aligned}
$$


Note that for a non-degenerate Poisson structure $\pi_{b}=a(z-b) \partial_{z} \wedge \partial_{\theta},|b|>1$ the total Liouville volume is given by the same formula, $V(\pi)=\frac{2 \pi}{a} \ln \left|\frac{1+b}{1-b}\right|$.

Corollary 3. Let $T \in \mathbb{R}^{+}$and $V \in \mathbb{R}$. A Poisson structure $\pi \in \mathcal{G}_{1}\left(S^{2}\right)$ with the modular period $T$ and the regularized total volume $V$ is globally equivalent to the Poisson structure which in coordinates $(z, \theta)$ on $S^{2}$ is given by

$$
\pi(T, V)=\frac{2 \pi}{T}\left(z-\frac{e^{V / T}-1}{e^{V / T}+1}\right) \partial_{z} \wedge \partial_{\theta}
$$

and vanishes linearly on the circle $z=\frac{e^{V / T}-1}{e^{V / T}+1}$.

Utilizing Poisson cohomology, D. Roytenberg [9] has previously obtained that the structures $\pi_{b},-1<b<1$ are non-trivial infinitesimal deformations of each other. Similarly, he proved that for each $b, \pi_{b}$ admits no infinitesimal rescalings. Using Theorem 3 , we get the following improvement of his results:

\section{Corollary 4.}

(a) The Poisson structures $\pi_{b}$ and $\pi_{b^{\prime}}$ are globally equivalent iff $b=b^{\prime}$.

(b) For $\alpha \in \mathbb{R} \backslash\{0\}$, the Poisson structures $\pi_{b}$ and $\alpha \pi_{b}$ are equivalent via an orientation-preserving Poisson isomorphism (respectively, arbitrary Poisson isomorphism) if and only if $\alpha=1$ (respectively, $|\alpha|=1$.) In particular, $\pi_{b}$ admits no rescalings.

\section{References.}

[1] V. Arnold, Mathematical methods of classical mechanics, Graduate Texts in Mathematics, 60, Springer-Verlag, New York, 1978.

[2] J.-P. Dufour and A. Haraki, Rotationnels et structures de Poisson quadratiques, C.R. Acad. Sci. Paris, 312 (1991), 137-140.

[3] M. Golubitsky and V. Guillemin, Stable mappings and their singularities, Graduate Texts in Mathematics, 14, Springer-Verlag, New York, 1973.

[4] J. Grabowski, G. Marmo, and A.M. Perelomov, Poisson structures: towards a classification, Modern Phys. Let., A 8(18) (1993), 1719-1733. 
[5] Z.-J. Liu and P. Xu, On quadratic poisson structures, Lett. Math. Phys., 26 (1992), 33-42.

[6] P. Monnier, Poisson cohomology in dimension 2, preprint, math.DG/ 000526 .

[7] J. Moser, On the volume elements on a manifold, Trans. AMS, 120(2) (1965), 286-294.

[8] J. Munkres, Elementary differential topology, Princeton University Press, 1963.

[9] D. Roytenberg, Poisson cohomology of SU(2)-covariant "necklace" Poisson structures on $S^{2}$, J. of Nonlinear Math. Phys., 9(3) (2002), 347-356.

[10] I. Vaisman, Lectures on the geometry of Poisson manifolds, Birkhäuser Verlag, Basel, 1994.

[11] A. Weinstein, The local structure of poisson manifolds, J. Differential Geom., 18 (1983), 523-557.

[12] A. Weinstein, The modular automorphism group of a poisson manifold, J. Geom. Phys., 23 (1997), 379-394.

[13] A. Weinstein, Poisson geometry, Diff. Geom. Appl., 9 (1998), 213-238.

Department of Mathematics

UCLA, Los Angeles, CA 90095 